Revue des patrimoines

$40 \mid 2019$

Lits historiques. Première anthologie des lits européens du XVe au XIXe siècle

\title{
Le vocabulaire et les typologies des lits en France au XVIII ${ }^{\mathrm{e}}$ siècle
}

Bed Vocabularies and Typologies in France during the Eighteenth Century

\section{Yves Carlier}

\section{(2) OpenEdition}

Journals

Édition électronique

URL : http://journals.openedition.org/insitu/23357

DOI : $10.4000 /$ insitu. 23357

ISSN : 1630-7305

Éditeur

Ministère de la Culture

Référence électronique

Yves Carlier, "Le vocabulaire et les typologies des lits en France au xvIII siècle », In Situ [En ligne], 40 | 2019, mis en ligne le 15 septembre 2019, consulté le 04 octobre 2019. URL : http://

journals.openedition.org/insitu/23357 ; DOI : 10.4000/insitu.23357

Ce document a été généré automatiquement le 4 octobre 2019.

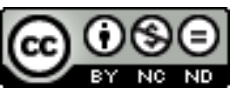

In Situ Revues des patrimoines est mis à disposition selon les termes de la licence Creative Commons Attribution - Pas d'Utilisation Commerciale - Pas de Modification 4.0 International. 


\title{
Le vocabulaire et les typologies des lits en France au XVIII ${ }^{\mathrm{e}}$ siècle
}

\author{
Bed Vocabularies and Typologies in France during the Eighteenth Century
}

Yves Carlier

1 Compris entre le long règne de Louis XIV et la chute de l'Ancien Régime, le siècle des Lumières connut une multiplication d'ouvrages visant à classer, rationnaliser et diffuser la connaissance. Des publications à portée généraliste comme les dictionnaires et l'Encyclopédie, des traités techniques comme ceux de Roubo ou Bimont, apportèrent chacun des définitions au mot lit, à ses composants et à ses différentes appellations ${ }^{1}$. Depuis, d'autres ouvrages ont tenté d'apporter des explications à ces termes, sans pour autant lever tous les doutes que l'on peut avoir à la lecture des documents anciens ${ }^{2}$.

De leur côté, les sources d'archives sont beaucoup plus riches et surtout plus variées : actes notariés (particulièrement les inventaires après décès), papiers du Garde-Meuble de la Couronne (dont en particulier les enregistrements des meubles consignés dans le Journal du Garde-Meuble ${ }^{3}$ et les mémoires des fournisseurs sur plus de vingt ans, de 1765 à 1792), papiers séquestrés durant la Révolution ${ }^{4}$. Tout ceci forme une masse non négligeable qui apporte des informations essentielles sur le vocabulaire employé durant le XviII ${ }^{\mathrm{e}}$ siècle. En parallèle, des recueils gravés issus de l'imagination féconde des ornemanistes ont multiplié les représentations du lit sous pratiquement toutes ses formes et sous toutes ses appellations ${ }^{5}$.

Pourtant, force est de constater qu'en de trop nombreuses occasions, la confrontation de ces documents ne permet pas toujours d'avoir une idée claire sur le pourquoi d'une appellation ni sur la raison de l'emploi d'un terme à la place d'un autre. À cause de la disparité du vocabulaire des uns et des autres (clercs de notaires, priseurs, experts nommés dans les inventaires, artisans, employés du Garde-Meuble) mais également des évolutions au long du siècle, les dénominations pouvaient varier selon les sources, voire être en contradiction, et ce sans compter celles parfois fantaisistes des ornemanistes ou celles dictées par la mode du moment. Ainsi, on se perd en conjectures sur la raison exacte - si jamais il y en eut une - de l'emploi de tel mot à la place de tel autre ou, 
inversement, on se demande si le terme utilisé par l'un l'aurait été différemment par un autre.

4 On constate également la disparition de mots dans le vocabulaire courant. Bien que le duc de Luynes l'ait employé pour décrire un nouveau lit de la reine à Versailles en 1743, le terme de quenouille (une colonne $)^{6}$ devient rare dans les actes notariés plus le siècle avance et n'apparaît jamais dans les documents du Garde-Meuble ${ }^{7}$.

\section{Couchette et lit}

5 Les mots couchette et lit (ou bois de lit) étaient employés de manière indifférenciée pour désigner la structure sur laquelle on s'allongeait. Elle se composait de deux chevets ou dossiers reliés entre eux par des éléments allongés nommés pans ou soubassements. Portés par des pieds, les chevets étaient soit intégralement couverts d'étoffe, soit intégralement en bois, soit composés d'un châssis mobile garni d'étoffe inséré dans un cadre. On continua pendant tout le siècle à mentionner des lits à bas piliers et à colonnes (ou piliers), termes remontant au siècle précédent (voir le texte de Nicolas Courtin). Le coucher reposait soit sur un châssis mobile sanglé sur lequel était posé un faux fond de toile, soit sur une structure en bois composée de goberges ${ }^{8}$ (parallèles aux chevets) et barres d'enfonçure (perpendiculaires aux chevets). La présence de roulettes confectionnées dans du gaïac, de l'acacia ou du buis selon l'importance du lit, devint de plus en plus fréquente au fil du temps. Montées sur pivots la plupart du temps, elles facilitaient le déplacement de lits d'un poids certain ou enfoncés dans des alcôves. Les gens de service et les personnes les plus humbles couchaient sur de simples lits de sangles composés d'un cadre formant châssis entre les montants desquels étaient clouées des sangles tendues et entrecroisées.

6 Le coucher proprement dit se composait d'éléments qui n'avaient guère évolué depuis le XVII ${ }^{e}$ siècle : un sommier de crin (qui n'est pas obligatoire), un ou des matelas de laine et futaine, un lit de plumes et coutil (c'est-à-dire un matelas peu épais), un ou des traversins ou oreillers de plumes et coutil ou de duvet et basin (coton), parfois couverts d'une "souille» (taie) en taffetas. Les couchers les plus rustiques étaient constitués d'une paillasse formée d'une toile épaisse bourrée de paille (de seigle de préférence).

7 Les lits se divisaient en deux catégories principales : ceux à chevet simple ou à double chevet dont le chevet principal (la tête) était parallèle au mur (nommés ci-après lit de bout) et ceux, systématiquement à deux chevets, dont les chevets étaient perpendiculaires au mur (nommés ci-après lit de travers).

\section{Pavillon, châssis, ciel, impériale ou baldaquin ?}

8 Plusieurs noms servaient à désigner la structure suspendue au plafond ou soutenue par des colonnes qui dominait la couchette et de laquelle pendaient les rideaux : pavillon, châssis, ciel, impériale, baldaquin. Parmi eux, certains ne posent pas de problème de compréhension mais d'autres sont moins évidents à interpréter, si jamais ils peuvent l'être au milieu des multiples informations contradictoires les concernant.

9 - Plus petit que la couchette qu'il surmonte, le pavillon est de forme circulaire et ses rideaux forment tente (fig. 1). L'emploi du mot était généralement associé aux lits destinés au personnel (selon l'Encyclopédie, «Les pavillons ne sont guère en usage que 
pour les lits de valets ») mais on le trouve parfois associé à des lits de personnages de haut rang.

Figure 1

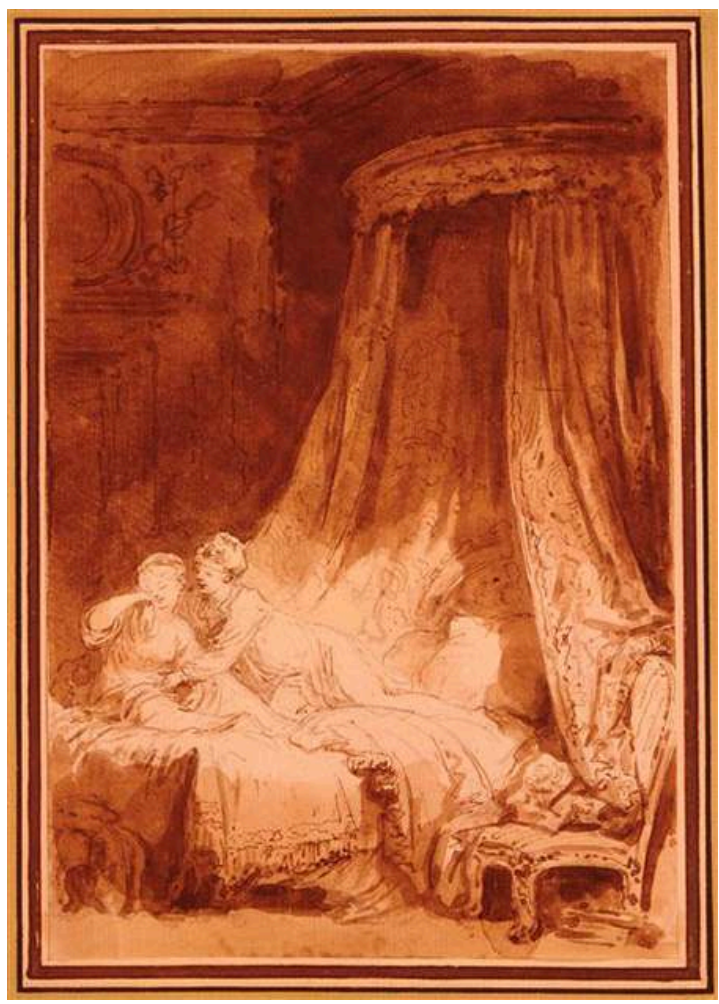

Jean-Honoré Fragonard, La courtisane amoureuse, Illustrations pour les contes et nouvelles de Jean de La Fontaine, 1765-1777. Dessin au lavis et à la pierre noire, Paris, Petit Palais, musée des Beaux-Arts de la Ville de Paris, L. Dut.1173.

(c) RMN-Grand Palais / Agence Bulloz.

10 - Le châssis (à ne pas confondre avec celui sanglé d'une couchette mentionné plus haut) est l'élément sur lequel sont attachées les étoffes. Il peut servir seul et possède dans ce cas des bords moulurés. Il peut aussi être l'un des composants d'une structure plus recherchée comme une impériale. Il peut rester plat ou être bombé et dans ce cas, il n'est pas rare de trouver le mot calotte ou châssis en dôme.

11 - Le terme de ciel ou ciel de lit semble avoir deux définitions. La première, très globale, fournie par les dictionnaires de Furetière ou Trévoux, est simplement le « haut d'un lit ». Il semble que c'est ce sens que retinrent les rédacteurs de l'inventaire après décès du marquis de Marigny, dans lequel le mot revient régulièrement pour différentes formes de lits, avec parfois les précisions «ciel de lit en baldaquin», "ciel en impériale $"$. L'autre définition, qu'illustrent plusieurs légendes des gravures de l' Encyclopédie, restreint le ciel à la seule partie en étoffe regardant la couchette. C'est manifestement ce sens qui a été retenu dans l'inventaire du duc de Brissac: un «baldaquin à ciel» ou un «baldaquin composé de son ciel $»^{10}$. Le dictionnaire de Furetière ajoute qu'il est préférable d'employer le mot fond à celui de ciel, et c'est le mot fond que l'on retrouve dans les documents du Garde-Meuble pour désigner cette partie en étoffe regardant la couchette. 
12 - La principale interrogation concerne la distinction qui était faite entre l'impériale et le baldaquin, deux mots qui devaient avoir à l'origine une signification précise, hélas absente des dictionnaires et auteurs contemporains. Ainsi, le Dictionnaire portatif des mots français (1750) définit-il l'impériale comme le sommet d'un lit et le baldaquin comme un ornement en forme de dais servant ordinairement à couronner un lit. De son côté, l'Encyclopédie considère l'impériale comme le châssis d'un lit mais malheureusement, ne possède pas d'entrée au mot baldaquin, bien qu'il y ait un renvoi à ce mot dans l'entrée au mot dais. Ce n'est que plus tard qu'Henry Havard définit l'impériale comme un ciel de lit façonné en dôme et le baldaquin comme un ciel de lit se présentant par son grand côté, sans que l'on sache d'où étaient tirées de telles assertions. Les traités de Roubo ou de Bimont n'apportent aucune aide, le premier utilisant uniquement le mot impériale, même pour des lits dont on voyait le grand côté, tandis que le second, au moment de définir les bois entrant dans la composition des lits, parlait des "châssis, impériales ou baldaquins " sans pour autant préciser de quoi il retournait et s'il établissait une différence entre eux. On ne sait pas non plus ce qu'il faut retenir du mémoire du menuisier Pascal qui, en 1787, facturait des baldaquins dont le derrière était plus large que le devant ${ }^{11}$. Dans son article, Nicolas Courtin fait état de la définition donnée par Félibien reprise par Richelet ${ }^{12}$ qui définissait l'impériale comme un dôme en pointe possédant un profil en $\mathrm{S}$. Malheureusement, si on se réfère aux seules planches de l'Encyclopédie, des profils différents (en dôme ou en S) sont nommés baldaquins et les impériales sont plates! Il ressort tout de même des textes que l'impériale devait le plus souvent être apparentée à un châssis offrant un profil élevé en son centre, comme le montrent les multiples estampes proposées par les ornemanistes $^{13}$ (fig. 2). 
Figure 2

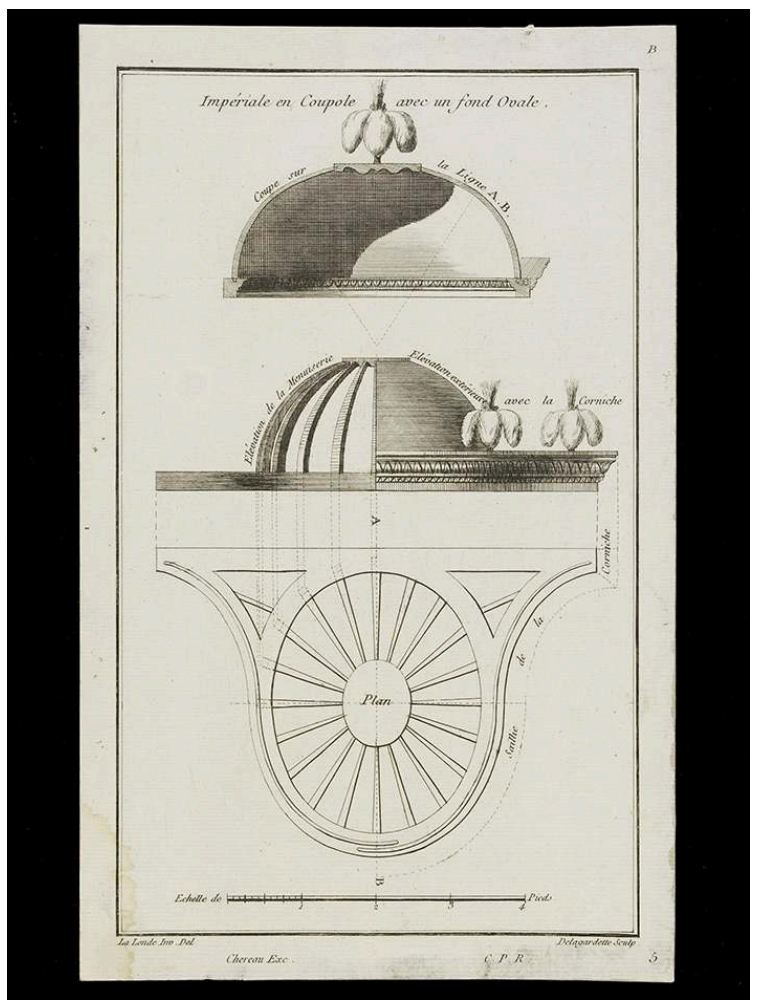

«Impériale à couple avec fond ovale », Richard de Lalonde, Cahiers d'ameublement, Delagardette (graveur). Paris : 1775-1782, pl. 5, Londres, Victoria and Albert Museum, E.33-1915.

(c) Victoria and Albert Museum. années 1750, le Garde-Meuble enregistrait des lits de composition similaire en leur accolant soit le nom de lits "en baldaquin", soit celui de lits "à impériale ", mais employa également l'expression de lit à "impériale en baldaquin ${ }^{14}$. Cette même expression, "impériale en baldaquin ", apparait dans un mémoire de Jean-Baptiste Boulard ou chez Nicolas-Quinibert Foliot pour des lits à la polonaise ${ }^{15}$.

Il ressort cependant des différents documents que le mot «impériale » était souvent utilisé comme synonyme de ciel de lit au sens global tandis que celui de baldaquin pouvait désigner un ciel d'une forme particulière, peut-être en dôme d'une verticalité plus accentuée. Pour simplifier les définitions des différentes formes de lit qui suivront, le mot d'impériale sera utilisé préférentiellement pour nommer la structure disposée au-dessus du lit.

- Dans les mémoires des menuisiers, sculpteurs et peintres-doreurs, le mot couronnement désigne la partie en bois sculpté ceinturant l'impériale et qui est indépendante du châssis garni d'étoffe ${ }^{16}$. On rencontre parfois à sa place le mot de corniche et, plus rarement, celui de bordure ${ }^{17}$.

\section{Les parties en étoffe}


18 - La courtepointe désigne la couverture plus ou moins épaisse pouvant inclure les traversins, ce qui correspond à nos actuels dessus de lit.

19 - Les soubassements sont les bandes d'étoffe recouvrant les côtés de la couchette. Le mot pente a parfois été employé à leur place, mais il est préférable de le conserver pour désigner l'habillement d'une impériale.

20 - Le chantourné correspond à la partie en étoffe disposée au niveau supérieur des chevets.

21 - Dans le cas d'une couchette, le mot dossier devrait être limité aux seules garnitures textiles couvrant les faces des chevets.

22 - Les fourreaux sont les garnitures en étoffe servant à gainer les colonnes ou piliers supportant les impériales.

23 Pour l'impériale :

24 - Le fond est la face de l'impériale garnie d'étoffe regardant la couchette.

25 - Le dossier ou grand dossier ne se retrouve pratiquement que dans le cas de lits de bout. Il s'agit de la partie en étoffe habillant l'espace entre l'impériale et le chevet du côté du mur.

26 - Les pentes sont les garnitures constituées de bandes d'étoffe accrochées aux bords de l'impériale et en faisant le tour. On distingue les pentes intérieures, au nombre de quatre, des pentes extérieures, au nombre de trois lorsque l'impériale est adossée au mur, et au nombre de quatre lorsqu'elle en est dégagée.

27 - Les rideaux sont de plusieurs sortes. Les bonnes grâces, que l'on trouve surtout dans les lits à colonnes ou les lits à la duchesse, sont placées au chevet de tête. Les cantonnières, qui ne se rencontrent théoriquement que dans les lits à colonnes, sont les rideaux disposés au chevet de pied. L'Encyclopédie ajoute qu'elles servent «à défendre l'entrée du vent qui pourrait venir par l'ouverture que laissent les grands rideaux ». Ces derniers, qui sont le plus souvent nommés tout simplement rideaux, sont montés sur anneaux coulissant sur tringle. Ce sont eux que l'on tire afin de préserver l'intimité et la chaleur dans la couche. Certains lits pouvaient de surcroît être pourvus d'une enveloppe supplémentaire, parfois nommée entour, tour de lit ou housse, également montée sur anneaux coulissant sur tringle qualifiée de « tournante ».

28 - Les pommes ou vases sont les éléments en ronde-bosse en bois intégralement couverts d'étoffe ou en bois sculpté dans les lits les plus luxueux, ornant les angles et, en quelques occasions, le sommet des impériales. Dans les lits les plus riches, ils recevaient une garniture de plumes d'autruche et d'aigrettes.

\section{Les incertitudes des appellations}

Les différentes appellations qui vont être abordées ci-dessous découlaient pour la plupart de la forme ou de la technique mise en œuvre pour la confection des étoffes. C'est le cas des lits à colonnes, à la duchesse, à la polonaise, à la romaine, en chaire à prêcher, à la turque, de campagne, en tombeau. Quelques appellations dérivaient de la fonction du lit (qui elle-même pouvait revêtir une forme particulière, comme le lit de veille) ou de son emplacement comme le lit en niche. Il faut garder à l'esprit qu'une dénomination n'implique pas un emplacement déterminé, puisque des estampes d'après Nicolas Pineau montrent des lits à la duchesse dans des niches, et François 
de Neufforge des lits à la turque dans une situation similaire (fig. 3). Dépourvus de couverture et n'étant de ce fait pas destinés à dormir (sauf de manière inopinée), les lits de repos n'ont pas été retenus dans ce corpus. Enfin, tous les lits étaient en bois (noyer, hêtre et chêne principalement); toutefois des lits en métal, matière jugée plus hygiénique, firent leur apparition vers la fin de l'Ancien Régime.

Figure 3

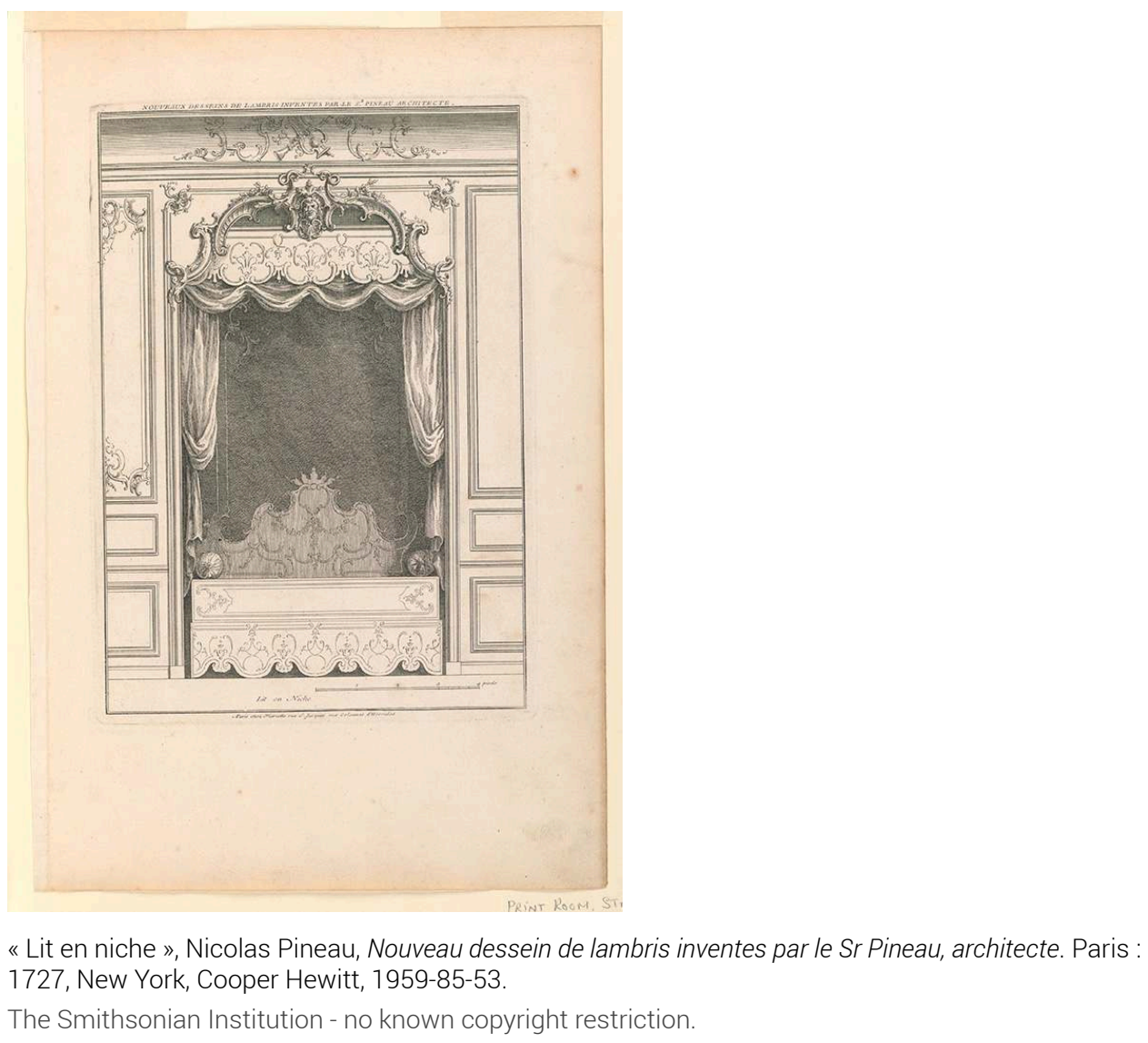

\section{Lit à colonnes}

La forme générale n'a pratiquement pas évolué depuis le XVII siècle (voir le texte de Nicolas Courtin). Il s'agit d'un lit de bout à un chantourné placé contre le mur que flanquent aux angles quatre colonnes supportant l'impériale qui est de la dimension de la couchette. On aimerait tout de même savoir pourquoi le Garde-Meuble nommait lits à colonnes des lits qu'il décrit comme pourvus de deux fourreaux de piliers ou deux colonnes de tête seulement ${ }^{18}$.

Cette même institution nommait lits à colonnes des lits à quatre colonnes et deux chantournés doubles, c'est-à-dire aux chevets garnis sur leurs deux faces, impliquant que ces lits à colonnes étaient des lits de travers comme on peut en voir un sur une miniature représentant une chambre du duc de Choiseul ${ }^{19}$. C'est probablement à cause d'une variante dans la forme de l'impériale que l'on retrouve, toujours au GardeMeuble, des lits à quatre colonnes et deux chevets dénommés à "impériale en cintre " ou à « impériale en dôme » (voir ci-dessous lit à impériale) ${ }^{20}$. 


\section{Lit à la duchesse}

Dérivant du lit à colonnes et anciennement nommé lit d'ange (voir le texte de Nicolas Courtin), il est également à un chantourné mais est dépourvu des deux colonnes du pied, ce qui permet de bien dégager son avant et de mieux le mettre en valeur par le retroussis des rideaux. L'impériale est alors soutenue par deux colonnes au chevet de tête ou maintenue au mur par des grandes tringles dites à oil. Durant les dernières décennies du siècle, il n'est pas rare de voir dans les grandes demeures l'ajout d'une colonne amovible disposée au pied du lit, dite colonne de pied, visant à renforcer le soutien de l'impériale durant la nuit (fig. 4).

Figure 4

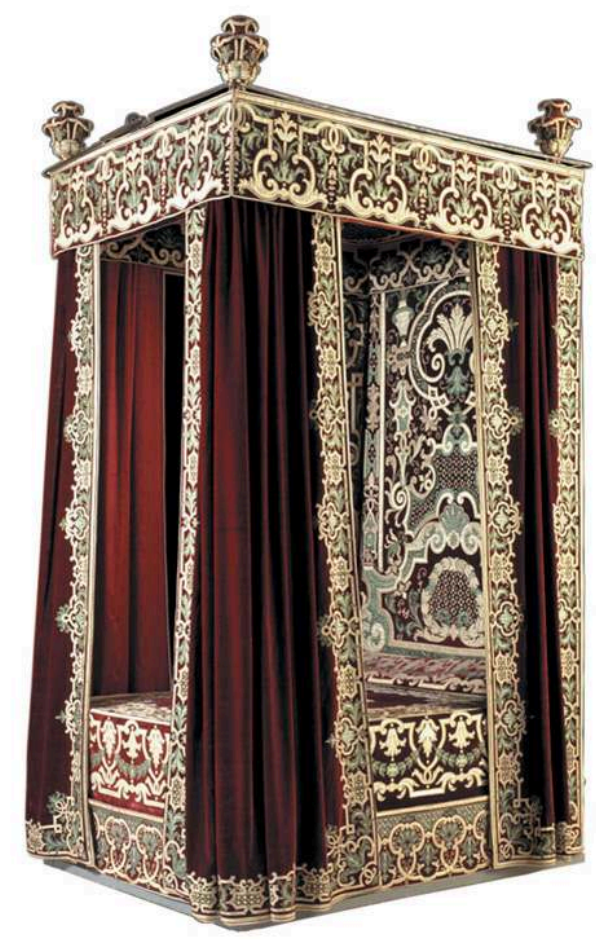

France, Lit de l'ambassadeur de Suède à la cour de Louis XIV, Nils Bielke, vers 1680. Stockholm, Nationalmuseum, NMK 1/1914:1.

(c) Nationalmuseum, Stockholm.

\section{Lit à impériale}

Ce terme apparaît régulièrement dans les papiers du Garde-Meuble et on ne sait s'il correspondait aux châssis ou housses «en impérial» inventoriés chez Mme de Pompadour dont la composition n'est pas assez détaillée ${ }^{21}$. L'analyse de ceux livrés au Garde-Meuble et des mémoires des fournisseurs montre que ces lits à impériale pouvaient être de deux sortes : lits à la duchesse ou lits à colonnes. Dans le premier cas, un lit «à impériale et voussure » enregistré en 1766 était bien décrit comme un lit à la duchesse par son auteur, le menuisier Nicolas-Quinibert Foliot ${ }^{22}$. De leurs côtés, les lits à « impériale en dôme » ou à « impériale en cintre», qui étaient tous à huit pentes, 
correspondaient à des lits à quatre colonnes et deux chevets, tels ceux enregistrés la même année que le même menuisier décrivit comme lits à quatre colonnes ${ }^{23}$.

Les impériales elles-mêmes pouvaient connaître des variantes d'appellation dues à une forme particulière qu'il est bien problématique de se figurer. En une occasion, le GardeMeuble inscrivit une «impériale à la chinoise " pour une calotte à huit pentes, nom devant refléter une forme peut-être inspirée de modèles anglo-chinois ${ }^{24}$. La même institution avait enregistré quelques lits à quatre colonnes et deux chevets sous le terme de « lit à colonnes et impériale à l'allemande » (ou bombé à l'allemande) ${ }^{25}$ et, à partir du règne de Louis XVI, des impériales "à la Choisy " (ou des lits «à la Choisy ») pour des lits à quatre colonnes et à un ou deux chevets ${ }^{26}$. Cette forme à la Choisy devait être bien reconnue puisque le terme était employé par tous les fournisseurs du GardeMeuble (menuisiers, sculpteurs, peintres, doreurs ou tapissiers) et apparaît même dans des mémoires non destinés à cette institution ${ }^{27}$.

\section{Lit à la romaine}

Apparu au cours du XVII ${ }^{e}$ siècle (voir le texte de Nicolas Courtin), cette typologie de lit perdura en quantité restreinte pendant le xviII ${ }^{\mathrm{e}}$ siècle, seule une dizaine de «lits à la romaine » étant recensée au Garde-Meuble jusque vers le milieu du siècle. Il s'agissait majoritairement de lits de travers à deux chevets, certains d'entre eux à quatre colonnes, et impériale dégagée puisqu'à huit pentes ${ }^{28}$. Ceci va à l'encontre de Bimont pour qui l'impériale du lit à la romaine est à trois faces, mais va dans le sens de la planche de l'Encyclopédie où elle est à quatre faces soutenue par quatre colonnes et quatre courbes en $\mathrm{S}$, proche en cela du lit à la polonaise ${ }^{29}$ (fig. 5). Seule sa forme en pavillon différait de celle du lit à la polonaise. 


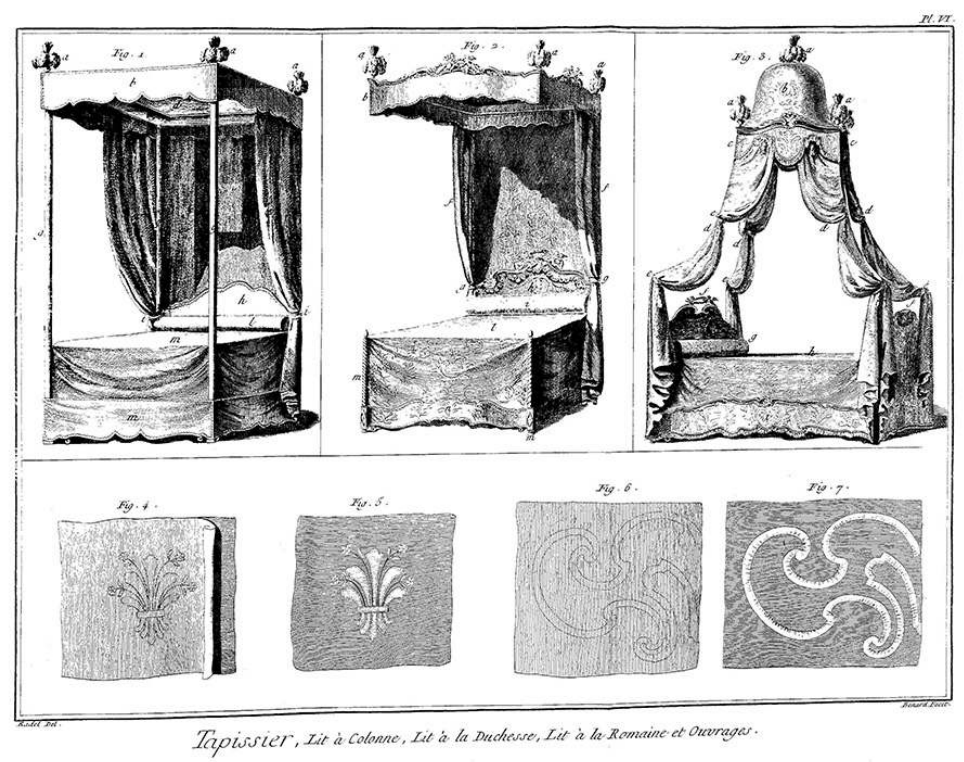

"Tapissier, Lit à la Colonne, Lit à la Duchesse, Lit à la Romaine et ouvrages », Denis Diderot et Jean Le Rond D'Alembert, Encyclopédie ou Dictionnaire raisonné des sciences, arts et métiers. Paris : Panckoucke, Stoupe et Brunet, 1777-1779.

(c) DR.

Par la suite, on a l'impression qu'une distinction a été établie entre le lit à la romaine et ce que les contemporains nommaient plus souvent baldaquin qu'impériale (le chapitre de Bimont s'intitule «lit à la romaine qu'on appelle Baldaquin »), qui paraît d'une dimension inférieure à la couchette. De surcroît, il n'est pas certain que l'un ait été systématiquement associé à l'autre. La fluctuation du vocabulaire et la dissociation entre couchette et impériale ou baldaquin à la romaine est sensible dans les mémoires des menuisiers.

Ce que Jacob nommait bois de lit à la romaine s'applique à des lits de travers à deux chevets et sans colonnes hautes. Il associait la forme avec différentes sortes d'impériales : impériale, châssis à moulures ou baldaquin à la romaine ${ }^{30}$.

Quant à la partie supérieure, on trouve aussi bien l'expression de «baldaquin à la romaine » chez Georges Jacob (voir note 28) que d'« impériale à la romaine », associée par Jean Avisse à un lit à la polonaise ou à une simple couchette à deux chevets, ou que Georges Jacob appliquait à un "lit fait en chaire à prêcher "31. De son côté, JeanBaptiste Claude Sené facturait une "impériale en baldaquin à la romaine " pour un lit que le Garde-Meuble enregistra comme «à la turque » alors que rien n'évoquait cette forme (voir ci-dessous lit à la turque).

\section{Lit en chaire à prêcher}

Ce terme n'apparaît pas avant les dernières années de l'Ancien Régime et se lit plus fréquemment dans les documents des périodes suivantes. Il désigne plus une impériale plaquée au mur qu'un lit dont la forme n'est pas sans évoquer celle d'un lit à la romaine (le Mobilier domestique l'associe au lit à la romaine ainsi qu'au lit à la d'Artois qui n'existe 
que chez les ornemanistes), mais pourvu de colonnes à l'arrière soutenant l'impériale comme l'indiquent clairement plusieurs mémoires de menuisiers ${ }^{32}$. Les impériales accompagnant ces lits étaient manifestement plus petites que la couchette, mais leur forme n'est pas fixe. Beaucoup de celles décrites dans les années 1790 et suivantes étaient à pans coupés ou à décrochement. Avant, Jacob en mentionnait une « cintrée en plan » et Boulard une " ceintrée des trois côtés $»^{33}$.

\section{Lit à la polonaise}

Ce nom apparaît pour la première fois au Garde-Meuble en 1765, mais il est très probable que cette forme était déjà bien établie car en 1750, le Garde-Meuble enregistrait sans lui accoler un nom spécifique un lit répondant à la description d'un lit à la polonaise (fig. 6). Sachant que ce lit était destiné à la reine, il y a de fortes présomptions que cette forme ait pris ce nom en raison des origines de Marie Leszczynska ${ }^{34}$. Le lit à la polonaise est un lit de travers à deux chevets surmonté d'une impériale dont Roubo précise qu'elle doit être d'un tiers plus petite que la couchette (toutefois celle figurant dans l'Encyclopédie est de même dimension que la couchette...). L'impériale est portée par quatre courbes en fer formant un $\mathrm{S}$ insérées dans les quatre colonnes qui cantonnent les coins de la couchette. Les rideaux sont souvent attachés à ces courbes de manière à les relever, dégager le lit et habiller les colonnes. En règle générale, les impériales des lits à la polonaise sont de forme ovale (toutefois Georges Jacob en mentionnait "de plan carré extérieur ${ }^{35}$ ) et dégagées du mur. Les lits à la polonaise ont par conséquent huit pentes, comme les lits à la romaine décrits par le Garde-Meuble ou celui figuré dans l'Encyclopédie. 
Figure 6

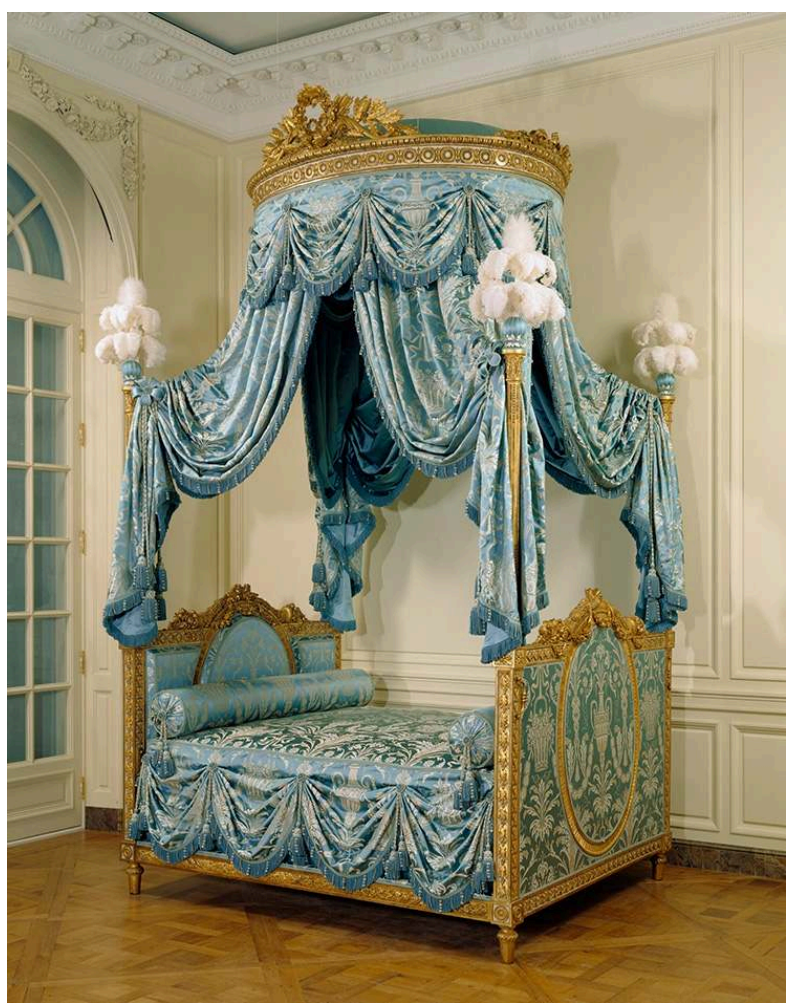

Paris, Lit à la polonaise, 1775-1780. Los Angeles, The J. Paul Getty Museum, 94.DA.72.

DigITAL IMAgE COURTESY OF THE GETTY'S OPEN CONTENT PROgRAM.

41 Le texte de Roubo ou une planche de Jean-Charles Delafosse prêtent à confusion puisqu'ils affirment qu'un lit à la polonaise doté d'un troisième chevet disposé le long du mur se nomme lit à l'italienne et non lit à la turque comme d'autres auteurs contemporains purent l'écrire.

\section{Lit à la turque}

L'apparition de ce qualificatif pour des lits de travers paraissant dériver des lits à la polonaise ne semble pas remonter avant le milieu du siècle (1764 au Garde-Meuble, qui mentionne un «bois de lit à la turque " pour un lit en niche $\left.{ }^{36}\right)$. Le terme recouvrait manifestement deux définitions qui pouvaient se conjuguer (fig. 7). 
Figure 7

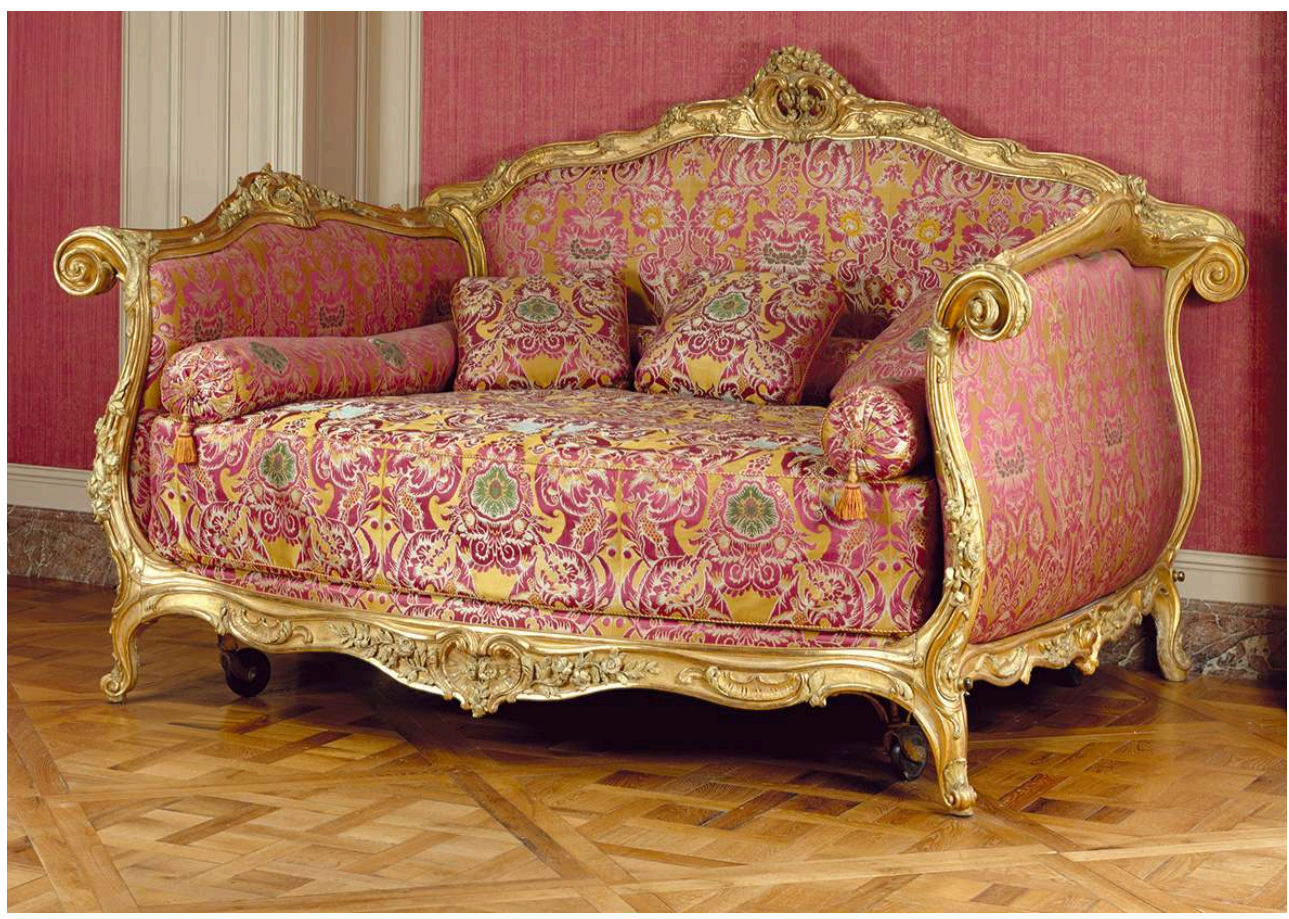

Jean-Baptiste Tilliard (attribué à), Lit à la turque, 1750-1760. Los Angeles, The J. Paul Getty Museum, 85.DA.535.

DIgITAL IMAgE COURTESY OF THE GETTY'S OPEN CONTENT PROgRAM.

La première, donnée par Bimont, est celle d'un lit à trois dossiers : un à la tête, un au pied et un dernier en fond de lit s'emboitant dans les deux autres ou pouvant se fixer au mur. Delanois livrait ainsi au comte d'Orsay deux bois de lit «à la turc» à trois dossiers ${ }^{37}$. Il faut toutefois préciser que cette forme est bien antérieure à l'apparition du terme puisque le Garde-Meuble enregistrait en 1734 l'entrée d'un lit en niche à trois chantournés sans pour autant lui attribuer un nom particulier ${ }^{38}$.

Figurant dans Roubo, la seconde définition est celle d'un lit dont le haut des dossiers du chevet et du pied se termine en enroulement parfois nommé «en crosse ». C'est ce qu'indiquent clairement François Foliot pour un lit à la turque avec rosaces dans le haut des crosses, Nicolas-Quinibert Foliot pour un lit à quatre colonnes avec des " crosses à la turque », ou le menuisier Pascal pour un lit à la turque à deux dossiers en crosse $^{39}$. A contrario, le Garde-Meuble enregistra en 1768 un lit à deux dossiers en crosse qu'il qualifie de «à la polonaise » et non de «à la turque » ${ }^{40}$.

45 L'association des deux définitions est représentée par le lit à la turque gravé dans l' Encyclopédie, qui comprend trois dossiers, ceux des chevets formant enroulement. On rencontre parfois des lits appelés "à la turque » sans qu'aucun des deux éléments caractérisant la forme de la couchette ne s'y retrouve, tel le lit de Louis XVI à SaintCloud, qui ne possédait que deux chevets verticaux sans enroulement ${ }^{41}$.

Ces lits étaient dotés d'une impériale placée le long du mur, donc à sept pentes, que Bimont signale plus riche que celle des lits à la romaine, bien que de même forme. 


\section{Lit en niche}

47 Même si Bimont considérait le lit en niche comme un lit de travers, les exemples fournis par les archives et les estampes prouvent que le terme recouvrait aussi bien des lits de cette catégorie que des lit de bout, à partir du moment où ils étaient disposés dans une alcôve ou une niche. On pouvait donc y placer des lits à la polonaise, à la turque, à la duchesse ou à colonnes. Tous se distinguaient des autres lits de même forme par la présence d'une seule pente extérieure et d'un seul soubassement, l'un et l'autre souvent nommés «de longueur ». Deux rideaux accrochés à une tringle fixée à l'intérieur de l'ouverture de la niche permettaient d'isoler la couchette.

\section{Lit à châssis en l'air}

Ce terme qui revient très régulièrement dans les papiers du Garde-Meuble désignait un lit fréquemment à un seul chevet surmonté d'un châssis simple, plat le plus souvent. Cette catégorie de lits garnis d'étoffe de qualité intermédiaire était généralement destinée au personnel domestique.

\section{Lit de veille}

Également destiné au personnel, le lit de veille est généralement un lit de sangle surmonté d'un pavillon circulaire d'où pend un rideau. L'immense majorité des pavillons et rideaux était de siamoise (étoffe de soie et coton) ou de serge et certains pouvaient être dotés d'une seconde enveloppe de toile. Dans la mesure où ces lits n'étaient déployés que pour la nuit, il est fréquent de lire que le bois était brisé, c'est-àdire monté avec des charnières pour pouvoir le plier et le serrer durant la journée. La possibilité de ranger les lits de service ou de libérer l'espace dans des maisons plus modestes existait dans d'autres types de lits comme le lit en armoire, que l'on rencontre au Garde-Meuble ou chez Mme de Pompadour ${ }^{42}$. Il s'agissait d'un lit de sangle à châssis brisé parfois accompagné d'un pavillon de serge, le tout se pliant dans une armoire. Dans le même genre figuraient les lits en coffre qui, dépourvus de pavillon, se pliaient et prenaient place dans un coffre en bois ${ }^{43}$.

\section{Lit en tombeau}

Garnissant souvent les pièces secondaires (ceux répertoriés par le Garde-Meuble se trouvaient dans les pièces de service ou les garde-robes), cette catégorie de lit pouvait être à simple ou à double tombeau. Dans le premier cas, les colonnes disposées à la tête du lit étaient plus hautes que celles du pied, induisant une inclinaison vers le pied de l'impériale, constituée de montants et traverses formant cadre. Cette dernière était ordinairement intégralement garnie d'étoffe. D'une forme proche du lit à la polonaise, le lit à double tombeau était à quatre colonnes de même hauteur portant une petite impériale formant double inclinaison. 


\section{Lit de campagne}

51 Sous ce terme se cachent les lits servant en voyage ou à l'armée, d'où le terme qui leur est accolé. Ils n'offraient donc pas de forme particulière mais devaient impérativement être pliants. Ainsi, les lits de campagne destinés au voyage de Marie-Anne-Victoire d'Espagne, l'Infante-Reine, éphémère épouse de Louis XV, étaient des lits à la duchesse dont il était précisé que les colonnes étaient brisées ${ }^{44}$.

52 La diversité des formes et appellations désignant les lits ne disparut pas avec la Révolution, comme en témoignent les procès-verbaux de saisies et de ventes dans lesquels ils figurent toujours. Les périodes suivantes continuèrent à innover tant en formes qu'en appellations nouvelles, pour le plus grand bonheur des tapissiers et des ornemanistes.

\section{NOTES}

1. - FURETIÈRE, Antoine. Dictionnaire universel contenant généralement tous les mots françois, [...] [1690]. Édition utilisée : La Haye : Husson, 1727 ; Dictionnaire universel françois et latin vulgairement appelé Dictionnaire de Trévoux [1704]. Édition utilisée : Paris : Compagnie des libraires associés, 1771 ; PRÉVOST, Claude Joseph et DUBOILLE, Charles. Manuel lexique, ou Dictionnaire portatif des mots françois dont la signification n'est pas familière à tout le monde [1750]. Paris: Didot, 1788; DIDEROT, Denis et ALEMBERT, Jean Le Rond d' (dir.). Encyclopédie, ou Dictionnaire raisonné des sciences, des arts et des métiers. Paris/Neuchâtel : 1751-1765, 17 vol. ; ROUBO, André-Jacob. L'Art du menuisier en meubles. Seconde section de la troisième partie de l'Art du menuisier. Paris : Saillant et Nyon, 1772 ; BIMONT, Jean-François. Principes de l'art du tapissier. Paris : Lottin l'Aîné, 1770.

2. - Ont été consultés: HAVARD, Henry. Dictionnaire de l'ameublement et de la décoration depuis le XIII siècle jusqu'à nos jours. Paris : Maison Quantin, [1887-1890], 4 vol. ; VERLET, Pierre. La Maison du XVIII ${ }^{e}$ siècle en France. Paris : Baschet, 1966 ; VERLET, Pierre. Les Meubles français du XVIII siècle [1956]. Paris : PUF, 1982 ; REYNIÈS, Nicole de. Le Mobilier domestique. Vocabulaire typologique. Paris : Imprimerie nationale, 1987, 2 vol.

3. - Conservé aux AN, série $\mathrm{O}^{1}$ (Maison du roi), 3304-3323 (1666-1790).

4. - AN, série T (Papiers privés tombés dans le domaine public).

5. - Ont été consultées les gravures des ornemanistes suivants: Juste-Nathan Boucher, JeanCharles Delafosse, Richard de Lalonde, Jean-François de Neufforge, Nicolas Pineau, Pierre Ranson. 6. - Voir dans ce numéro : BARBIER, Muriel. « Le vocabulaire des lits de la Renaissance en France à la lumière des sources ", In Situ [En ligne], 40 | 2019, mis en ligne le 10 septembre 2019, consulté le 30 septembre 2019. URL: http://journals.openedition.org/insitu/23340 ; COURTIN, Nicolas. «Le vocabulaire et les typologies des lits à Paris d'après les inventaires après décès de la première moitié du XVII siècle », In Situ [En ligne], 40 |2019, mis en ligne le 15 septembre 2019, consulté le 30 septembre 2019. URL : http://journals.openedition.org/insitu/23316.

7. - «Le lit n'est point à quatre quenouilles, comme tous les lits de la Reine ont été jusqu'à présent; il est ce qu'on appelle à la duchesse. " (LUYNES, Charles-Philippe d'Albert, duc de. Mémoires du duc de Luynes sur la Cour de Louis XV (1735-1758). Édition Louis Dussieux et Eudore Soulié. Paris : Firmin-Didot, 1860-1865, t. V, 1743-1744, 1861, p. 13). 
8. - Petites traverses de bois placées sur le fond d'un lit et qui servent à supporter le matelas (TLF, voir le site : http://atilf.atilf.fr/dendien/scripts/tlfiv5/advanced.exe?55;s=3425924235).

9. - GORDON, Alden R. The Houses and Collections of the Marquis de Marigny. Los Angeles: Getty Publications, 2003, passim.

10. - AN, MC/XCI/1295, 28 décembre 1792.

11. - Ibid., $\mathrm{O}^{1} 3641$. Dans un cas, le menuisier précise avoir réduit un baldaquin, son châssis et son couronnement et, dans un autre, avoir fourni un baldaquin pour un lit en chaire à prêcher.

12. - Voir dans ce numéro : COURTIN, Nicolas. «Le vocabulaire et les typologies des lits à Paris d'après les inventaires après décès de la première moitié du XVII ${ }^{\mathrm{e}}$ siècle », In Situ [En ligne], 40 | 2019, mis en ligne le 15 septembre 2019, consulté le 30 septembre 2019. URL: http:// journals.openedition.org/insitu/23316.

13. - On lit parfois des descriptions d'impériales doubles et en une occasion triples (chez le prince de Condé) qui correspondent à des châssis bombés superposés comme le montre une estampe de Lalonde.

14. - AN, $\mathrm{O}^{1} 3352, \mathrm{n}^{\circ} 798$.

15. - Ibid., T $208^{9} ; \mathrm{O}^{1} 3617$ et $\mathrm{O}^{1} 3621$.

16. - En 1784, le mot de couronnement est employé par les menuisiers Geoffroy, Jacob, Boulard et Foliot, les sculpteurs Rousseau et Foliot, les peintres-doreurs Bardou et Chatard (ibid., $\mathrm{O}^{1} 3629$ à $\left.\mathrm{O}^{1} 3631\right)$.

17. - Le mot de corniche se retrouve chez Nicolas-Quinibert Foliot en 1768 ou 1771 par exemple (ibid., $\mathrm{O}^{1} 3620$ et $\mathrm{O}^{1} 3623$ ) ou sur une estampe de Lalonde. De son côté, Georges Jacob l'employait plutôt pour désigner une moulure sculptée à l'intérieur de l'impériale (LEFUEL, Hector-Martin. Georges Jacob, ébéniste du XVIII siècle. Paris : A. Morancé, 1923, p. 152 et suiv.). Celui de bordure a été employé par Nicolas-Quinibert Foliot en 1770 (AN, $\left.\mathrm{O}^{1} 3622\right)$.

18. - AN, $\mathrm{O}^{1} 3314$, f. $42 \mathrm{v}^{\mathrm{o}}$ et $\mathrm{O}^{1} 3315$, f. $81 \mathrm{v}^{\mathrm{o}}$.

19. - Ibid., $\mathrm{O}^{1} 3317$, f. $172 \mathrm{v}^{\mathrm{o}}$.

20. - Ibid., $\mathrm{O}^{1} 3314$, f. $181 \mathrm{v}^{\mathrm{o}}$ et $\mathrm{O}^{1} 3319$, f. 12.

21. - Ibid., $\mathrm{O}^{1} 3312$, f. $169 \mathrm{v}^{\circ}$; CORDEY, Jean. Inventaire des biens de Madame de Pompadour : rédigé après son décès. Paris : Société des bibliophiles françois, 1939, p. 13 et 53.

22. - $\mathrm{AN}, \mathrm{O}^{1} 3318, \mathrm{f} .122 \mathrm{v}^{0}$ et $\mathrm{O}^{1} 3618$.

23. - Ibid., $\mathrm{O}^{1} 3318$, f. 123 et $\mathrm{O}^{1} 3618$.

24. - Ibid., $\mathrm{O}^{1} 3319$, f. 223.

25. - Ibid., $\mathrm{O}^{1} 3318$, f. $88 \mathrm{v}^{\circ}$ et $\mathrm{O}^{1} 3319, \mathrm{f} .100 \mathrm{v}^{\circ}$. Le menuisier Nicolas-Quinibert Foliot employa également cette terminologie (ibid., $\mathrm{O}^{1} 3621$ ).

26. - Le terme est employé la première fois par le serrurier du Garde-Meuble en 1776 (AN, $\mathrm{O}^{1}$ $3625^{1)}$, puis apparait régulièrement les années suivantes chez différents fournisseurs.

27. - LEFUEL, Hector-Martin. Op. cit., p. 188, 222, 231, 254, 266, 275...

28. - $\mathrm{AN}, \mathrm{O}^{1} 3313, \mathrm{f} .20 \mathrm{v}^{\mathrm{o}}$ et $91 \mathrm{v}^{\mathrm{o}} ; \mathrm{O}^{1} 3314, \mathrm{f} .148 \mathrm{v}^{\circ}$ et $149 ; \mathrm{O}^{1} 3316$, f. 122 . Les lits à quatre colonnes, ibid. $\mathrm{O}^{1} 3315$, f. $99 \mathrm{v}^{\circ} ; \mathrm{O}^{1} 3316$, f. $57 \mathrm{v}^{\circ}, 66 \mathrm{v}^{\circ}$ et $111 \mathrm{v}^{\circ}$.

29. - En 1755, un des Avisse avait confectionné une impériale à la romaine pour un lit à la polonaise (AN, T $186^{72}$ ).

30. - LEFUEL, H.-M. Op. cit., p. 220, 222, 249, 267, 272, 332.

31. - AN, T $186^{72}$; LEFUEL, H.-M. Op. cit, p. 242.

32. - Mémoire de Boulard en 1784 et de Pascal en 1787 (AN, $\mathrm{O}^{1} 3630$ et $\mathrm{O}^{1} 3641$ ).

33. - LEFUEL, H.-M. Op. cit., p. 242 ; AN, $\mathrm{O}^{1} 3630$.

34. - BONNET, Xavier. « Les tapissiers ordinaires du Roi (1666-1789) ». Versalia, $\mathrm{n}^{\circ}$ 20, 2017, p. 90.

35. - LEFUEL, H.-M. Op. cit., p. 223.

36. - AN, $\mathrm{O}^{1} 3318$, f. $51 \mathrm{v}^{\mathrm{o}}$.

37. - ERIKSEN, Svend. Louis Delanois, menuisier en sièges (1731-1792). Paris : F. De Nobele, 1968, p. 52.

38. - AN, $\mathrm{O}^{1} 3311$, f. 188. 
39. - Ibid., T $201^{101} ; \mathrm{O}^{1} 3617 ; \mathrm{O}^{1} 3641$.

40. - Ibid., $\mathrm{O}^{1} 3318$, f. 114.

41. - Qualifié de «à la turque » dans divers documents, il ne possédait que deux dossiers, comme le prouve le mémoire du tapissier (AN, $\left.\mathrm{O}^{1} 3648\right)$.

42. - Ibid., $\mathrm{O}^{1} 3317$, f. 159v $\mathrm{v}^{\circ}$; CORDEY, J. Op. cit., p. 23, 24, 114, 159 et 194.

43. - AN, $\mathrm{O}^{1} 3319, \mathrm{f} .104 \mathrm{v}^{\circ}$. Ces lits devaient s'apparenter aux lits en banquette mentionnés de manière épisodique.

44. - AN, $\mathrm{O}^{1} 3310$, f. 27.

\section{RÉSUMÉS}

Période de rationalisation, des encyclopédies et des dictionnaires, le xviiie siècle n'a pourtant pas vu le vocabulaire lié aux lits complètement normalisé. D'anciens termes hérités des siècles précédents continuent d'être utilisés, tandis que de nouveaux voient le jour alors qu'artistes et artisans usent de leurs propres habitudes langagières. À la lecture des sources (inventaires, mémoires des fournisseurs), la consultation des ouvrages de l'époque et l'observation des modèles des ornemanistes et des architectes, l'on constate la richesse mais aussi la fluctuance des termes employés par les différents métiers. La désignation des parties du bâti, la terminologie des parties en étoffes et les typologies nouvelles de lit varient en fonction des personnes. On ne peut que souligner les incertitudes des appellations liées à la forme des lits ou à la technique de confection des textiles. Pourtant, il demeure possible de proposer une lecture des dénominations rencontrées dans ces documents du xviiie siècle.

Although it was a period of rationalisation, of encyclopaedias and of dictionaries, the eighteenth century did not see a thorough-going normalisation of the vocabulary associated with beds. Old expressions inherited from earlier centuries continued to be used whilst new ones emerged and craftsmen and artists had their own language customs. Sources such as inventories, the bills of furniture suppliers and publications of the time and the observation of models proposed by decorators or architects all suggest something of the richness of the terms employed by different trades, but also their fluctuating meanings. The designation of the built parts of the bed frame, the terminology used for the textile components and the new bed types vary according to the user. But although it is necessary to underline the uncertainty that obscures the appellations associated with the shape of beds or the techniques of textile manufacture, it remains possible to propose a general understanding of the denominations to be found in these eighteenth-century documents.

\section{INDEX}

Keywords : Jean-François Bimont, furniture repository, bed, alcove, André-Jacob Roubo, eighteenth century, vocabulary, ornementalist, Jean-Baptiste Boulard, Nicolas-Quinibert Foliot, François Foliot, Nicolas Pineau, Jean-François de Neufforge, Louis Delanois

Mots-clés : Jean-François Bimont, garde-meuble, lit, alcôve, André-Jacob Roubo, XVIIIe siècle, dix-huitième siècle, vocabulaire, ornemaniste, Jean-Baptiste Boulard, Nicolas-Quinibert Foliot, François Foliot, Nicolas Pineau, Jean-François de Neufforge, Louis Delanois 
AUTEUR

YVES CARLIER

Conservateur général, Département de la gestion des collections, château de Versailles yves.carlier@chateauversailles.fr 